\title{
Early Prediction of Response to Chemotherapy and Survival in Malignant Pleural Mesothelioma Using a Novel Semiautomated 3-Dimensional Volume-Based Analysis of Serial ${ }^{18}$ F-FDG PET Scans
}

\author{
Roslyn J. Francis ${ }^{1}$, Michael J. Byrne ${ }^{2}$, Agatha A. van der Schaaf ${ }^{1}$, Jan A. Boucek ${ }^{1}$, Anna K. Nowak ${ }^{2,3}$, Michael Phillips ${ }^{4}$, \\ Richard Price ${ }^{5}$, Andrew P. Patrikeos ${ }^{1}$, A. William Musk ${ }^{6}$, and Michael J. Millward ${ }^{2,3}$ \\ ${ }^{I}$ Department of Nuclear Medicine/WA PET Centre, Sir Charles Gairdner Hospital, Nedlands, Western Australia, Australia; ${ }^{2}$ Department \\ of Medical Oncology, Sir Charles Gairdner Hospital, Nedlands, Western Australia, Australia; ${ }^{3}$ School of Medicine and Pharmacology, \\ Faculty of Medicine and Dentistry and Health Science, University of Western Australia, Western Australia, Australia; ${ }^{4}$ Cancer Council \\ Clinical Trials Biostatistics Department, WA Institute for Medical Research, Sir Charles Gairdner Hospital, University of Western \\ Australia, Nedlands, Western Australia, Australia; ${ }^{5}$ Department of Radiology, Sir Charles Gairdner Hospital, Nedlands, Western Australia, \\ Australia; and ${ }^{6}$ Department of Respiratory Medicine, Sir Charles Gairdner Hospital, Nedlands, Western Australia, Australia
}

The aim of chemotherapy for mesothelioma is to palliate symptoms and improve survival. Measuring response using CT is challenging because of the circumferential tumor growth pattern. This study aims to evaluate the role of serial ${ }^{18}$ F-FDG PET in the assessment of response to chemotherapy in patients with mesothelioma. Methods: Patients were prospectively recruited and underwent both ${ }^{18}$ F-FDG PET and conventional radiological response assessment before and after 1 cycle of chemotherapy. Quantitative volume-based ${ }^{18} \mathrm{~F}$-FDG PET analysis was performed to obtain the total glycolytic volume (TGV) of the tumor. Survival outcomes were measured. Results: Twenty-three patients were suitable for both radiological and ${ }^{18} \mathrm{~F}-\mathrm{FDG}$ PET analysis, of whom 20 had CT measurable disease. After 1 cycle of chemotherapy, 7 patients attained a partial response and 13 had stable disease on CT assessment by modified RECIST (Response Evaluation Criteria in Solid Tumors) criteria. In the 7 patients with radiological partial response, the median TGV on quantitative PET analysis fell to $30 \%$ of baseline (range, $11 \%-71 \%$ ). After 1 cycle of chemotherapy, Cox regression analysis demonstrated a statistically significant relationship between a fall in TGV and improved patient survival $(P=0.015)$. Neither a reduction in the maximum standardized uptake value $(P=0.097)$ nor CT $(P=0.131)$ demonstrated a statistically significant association with patient survival. Conclusion: Semiquantitative ${ }^{18} \mathrm{~F}-\mathrm{FDG}$ PET using the volume-based parameter of TGV is feasible in mesothelioma and may predict response to chemotherapy and patient survival after 1 cycle of treatment. Therefore, metabolic imaging has the potential to improve the care of patients receiving chemotherapy for mesothelioma by the early identification of responding patients. This technology may also be useful in the assessment of new systemic treatments for mesothelioma.

Received Apr. 5, 2007; revision accepted Jun. 8, 2007.

For correspondence or reprints contact: Roslyn Francis, MBBS, PhD, Department of Nuclear Medicine/WA PET Centre, Sir Charles Gairdner Hospital, Verdun St., Nedlands, Western Australia, 6009, Australia.

E-mail: Ros.Francis@health.wa.gov.au

COPYRIGHT @ 2007 by the Society of Nuclear Medicine, Inc.
Key Words: ${ }^{18}$ F-FDG PET; mesothelioma; response; chemotherapy

J Nucl Med 2007; 48:1449-1458

DOI: 10.2967/jnumed.107.042333

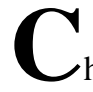

hemotherapy for patients with malignant pleural mesothelioma is predominantly palliative, with the aim of relieving symptoms, improving quality of life, and increasing survival. Early identification of responding patients is important so that chemotherapy can be continued in patients in whom the potential benefits outweigh toxicity. Patients who are not likely to benefit may, therefore, be spared the side-effects of further therapy.

The measurement of response to chemotherapy in pleural mesothelioma remains problematic. The "rindlike" pattern of growth around pleural surfaces provides few opportunities for bidimensional measurement of discrete lesions. A modification of the RECIST (Response Evaluation Criteria in Solid Tumors) criteria specifically for mesothelioma, based on the measurement of tumor thickness at multiple levels on CT scan, has partially addressed the problem (1). Using the modified RECIST criteria, a partial response (PR) correlates with relief of symptoms, an increase in forced expiratory volume, improved health-related quality of life, and patient survival $(2,3)$. However, the method is time-consuming and subject to errors, both in measurement and in assessing target tissue. It is also based on structure and takes no account of the viability of tumor masses. Finally, radiological assessment is an insensitive tool. In most cases it is not clear whether a tumor is responsive to chemotherapy until several cycles of treatment have been given. In a previous study we found that in only $47 \%$ of those who achieved a PR could this be detected after 1 cycle of therapy (2). 
Radiological response criteria document change in tumor size. However, uptake of tracer in ${ }^{18}$ F-FDG PET scans is a measure of the metabolic activity and, therefore, the viability of tumor cells. In several solid tumors, serial changes in tumor tracer uptake have been shown to predict response to chemotherapy after only 1 cycle of therapy (4-7).

Mesothelioma is ${ }^{18} \mathrm{~F}$-FDG avid (8) (Fig. 1), and initial studies suggest that ${ }^{18} \mathrm{~F}$-FDG PET may be useful in the assessment of prognosis and in staging of patients with this disease $(9,10)$. However, the role of ${ }^{18}$ F-FDG PET in the assessment of response to chemotherapy, particularly in a prospective setting, has not been clearly established.

There are no formalized guidelines for the measurement of response to chemotherapy using ${ }^{18} \mathrm{~F}$-FDG PET in solid tumors (11). Visual analysis is challenging, particularly in mesothelioma, because of the extent and distribution of disease. The most common semiquantitative parameter used is the maximum standardized uptake value (SUVmax) within a tumor (12-14). Mesothelioma is poorly suited to SUVmax measurements as it is often diffuse and heterogeneous. Defining the site to apply a representative SUVmax measurement is difficult and potentially unreliable. In addition, a measure that defines change on the basis of only 1 pixel within such a complex tumor mass is likely to be an oversimplification.

There is emerging interest in volume-based measures for response assessment with ${ }^{18} \mathrm{~F}-\mathrm{FDG}$ PET. Defining the tumor volume manually is laborious and unreliable. Therefore, we have developed novel software that semiautomatically defines the 3-dimensional (3D) boundaries of the tumor on ${ }^{18} \mathrm{~F}$-FDG PET scans. This results in a measure of the total glycolytic volume (TGV), which is a composite of tumor volume and total metabolic activity. We used this methodology to compare the response to 1 cycle of chemotherapy, as assessed by serial CT scans, with changes on serial ${ }^{18} \mathrm{~F}$-FDG PET scans in patients with malignant pleural mesothelioma.

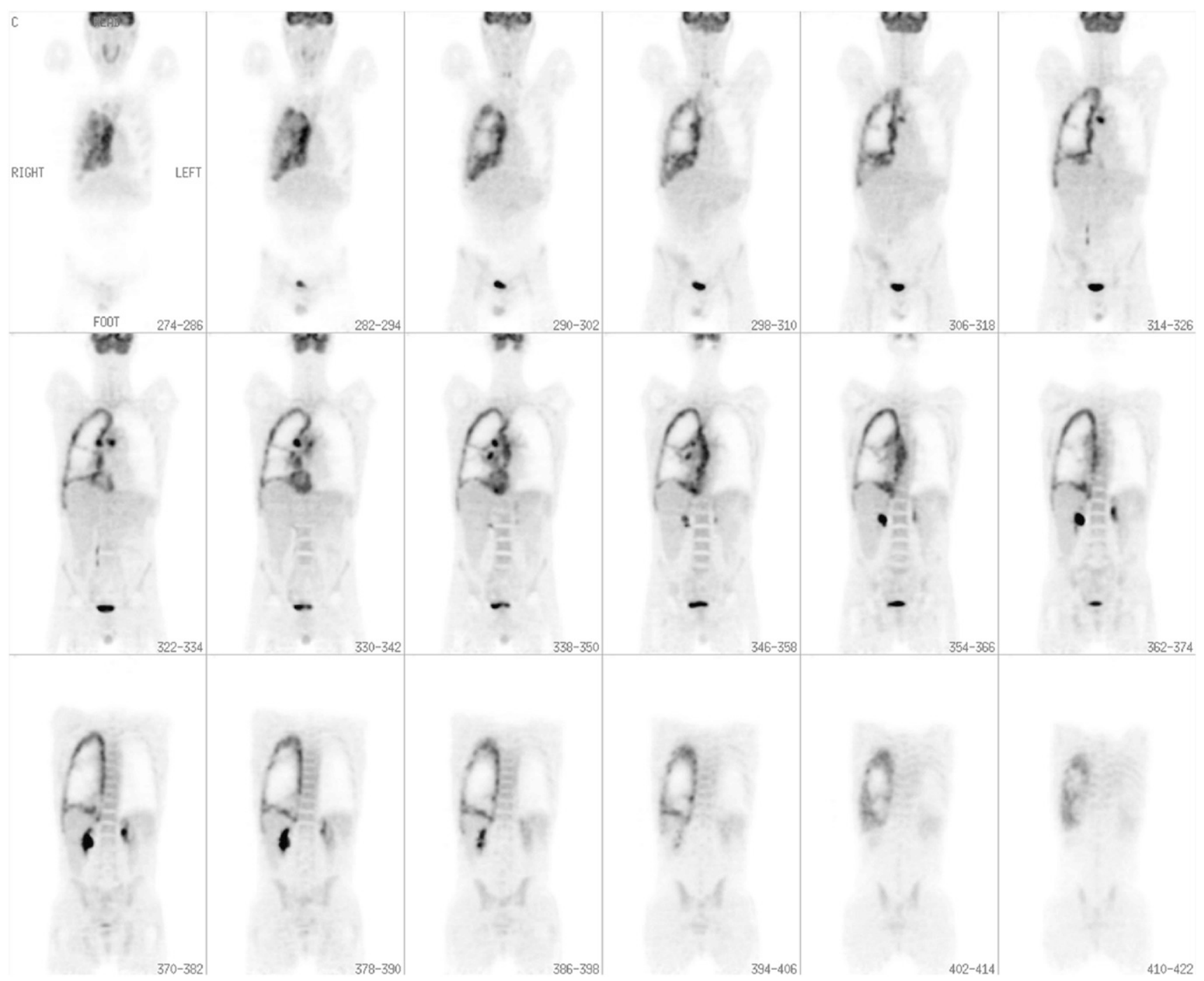

FIGURE 1. Coronal slices of an ${ }^{18} \mathrm{~F}-\mathrm{FDG}$ PET scan of a patient with mesothelioma demonstrating contiguous involvement of the right pleural surface, including infiltration of the oblique fissure. There is additional subcarinal, precarinal, right paratracheal, and right hilar lymph node involvement. 


\section{MATERIALS AND METHODS}

\section{Patients}

Patients with mesothelioma commencing chemotherapy as part of usual care were prospectively recruited to the study. Eligible patients had histologically or cytologically proven malignant pleural mesothelioma, no prior chemotherapy, age $>18$ y, European Cooperative Oncology Group performance status 0-2, and either CT measurable or nonmeasurable disease. All patients had adequate bone marrow, renal and hepatic function as indicated by the following: total leukocyte count, $>3 \times 10^{9} / \mathrm{L}$; granulocyte count, $>1.5 \times$ $10^{9} / \mathrm{L}$; platelet count, $>100 \times 10^{9} / \mathrm{L}$; hemoglobin, $>10 \mathrm{~g} / \mathrm{dL}$; serum creatinine, $<120 \mu \mathrm{mol} / \mathrm{L}$; total bilirubin, $<1.5 \times$ the upper limit of normal; alanine transaminase and alkaline phosphatase, $<3 \times$ the upper limit of normal. Patients with recent talc pleurodesis were initially recruited to the study; however, as published information became available on the confounding inflammatory effects of talc pleurodesis on ${ }^{18}$ F-FDG PET (15), the eligibility criteria were amended to exclude patients with talc pleurodesis performed within 6 mo before study entry.

All patients received chemotherapy with cisplatin $\left(100 \mathrm{mg} / \mathrm{m}^{2}\right.$ on day 1 ) and gemcitabine (1,000 mg/m $\mathrm{m}^{2}$ on days 1,8 , and 15$)$. Cycles were repeated at 28 -d intervals. Patients who had CT-measurable disease were scheduled to have 6 cycles of chemotherapy in the absence of disease progression or unacceptable toxicity. Those with CT-nonmeasurable disease were scheduled to have 4 treatment cycles.

\section{Schedule of Investigations}

Before the commencement of each cycle of chemotherapy patients had a clinical examination and assessment of full blood picture, serum creatinine, electrolytes, and liver function tests.

$C T$. Each patient had a contrast-enhanced thoracic and upper abdominal helical CT scan with 5-mm slices $<4$ wk before the first cycle of chemotherapy. This was repeated after the first and third or fourth cycle, with an extra CT scan to confirm response when appropriate.

CT Analysis. Radiological response to therapy was assessed using the modified RECIST criteria for mesothelioma (1). CT scans were assessed by a thoracic radiologist, experienced in mesothelioma measurement and unaware of patient outcome and ${ }^{18} \mathrm{~F}$ FDG PET scan results.

Complete response (CR) was defined as the disappearance of all target lesions with no evidence of tumor elsewhere, and PR was defined as at least a $30 \%$ reduction in the summated unidimensional tumor measurement. A confirmed response required a repeat observation on 2 occasions at least $4 \mathrm{wk}$ apart. Progressive disease (PD) was defined as an increase of at least $20 \%$ in the total tumor measurement over the nadir measurement, or the appearance of one or more new lesions. Patients with stable disease (SD) were those who fulfilled the criteria for neither PR nor PD.

${ }^{18}$ F-FDG PET. Patients had an ${ }^{18} \mathrm{~F}-\mathrm{FDG}$ PET scan performed $<4$ wk before commencing chemotherapy and after the first cycle of chemotherapy using a Phillips Allegro germanium oxyorthosilicate PET scanner. Patients fasted for $6 \mathrm{~h}$ and had a blood glucose level measured before ${ }^{18} \mathrm{~F}-\mathrm{FDG}$ administration. The blood glucose level was $<10 \mathrm{mmol} / \mathrm{L}$ in all patients. ${ }^{18} \mathrm{~F}-\mathrm{FDG}\left(200 \mathrm{MBq} / \mathrm{m}^{2}\right)$ was administered intravenously, and patients were imaged after $90 \mathrm{~min}$ of rest in a quiet room. Emission scans were performed over multiple bed positions, with 4 min per bed position and 50\% overlap per field of view. ${ }^{137} \mathrm{Cs}$ transmission scans were acquired for attenua- tion correction. Patient preparation, imaging, and reconstruction protocols were kept constant for serial scans in the same patient.

${ }^{18}$ F-FDG PET Analysis. Quantitative analysis was performed using our semiautomated 3D volume-based region-growing algorithm. The growing function of the algorithm is threshold-based and is determined by the current mean, the activity in neighboring pixels, and the maximum normal background level (Appendix) $(16,17)$. Contiguous 3D tumor regions are generated, with the operator reseeding on noncontiguous tumor elements. The reproducibility and accuracy of the algorithm has been validated in phantom simulations, and an interobserver variability of approximately 5\% for the measurement of TGV has been demonstrated in a clinical setting (17).

The region-growing algorithm was used to define a volume of interest (VOI) on both the pre- and posttherapy ${ }^{18} \mathrm{~F}-\mathrm{FDG}$ PET scans. The semiquantitative measures of TGV and SUVmax were obtained from the VOI. TGV is a composite of metabolic activity and volume within the tumor mass and, therefore, has the units of SUV.mL. SUVmax was regarded as the maximum SUV value in the tumor VOI.

TGV and SUVmax values after 1 cycle of chemotherapy were compared with the baseline measurements.

The decision to stop therapy because of progression was made using the modified RECIST criteria and on clinical assessment. The ${ }^{18}$ F-FDG PET scan results were not used to direct patient therapy.

\section{Ethical Considerations}

All patients received printed information regarding the study and gave written informed consent to participate. The appropriate Human Research Ethics Committee approved this study.

\section{Statistical Analysis}

Survival was calculated from day 1 of the first cycle of chemotherapy until death (from any cause). Cox regression analysis was the primary statistical test performed to assess the relationship between change in PET parameters or CT response and survival. Hazard ratios were calculated for a $10 \%$ change from baseline for measured $\mathrm{CT}$ and PET response parameters, as a $1 \%$ change is unlikely to have practical clinical significance for patient management and is very likely to be prone to error. Kaplan-Meier curves using incremental TGV response values of $>85 \%, 60 \%-85 \%$, and $<60 \%$ of baseline were used to illustrate survival differences between groups. Significance was regarded at the 5\% level. Statistical analyses were performed using SPSS 14.0 (SPSS Inc.) and Stata 9.3 (StataCorp LP).

\section{RESULTS}

Thirty-nine patients were prospectively recruited to the study over a 2-y period. Two patients who had synchronous second primary tumors and 8 who did not have a second CT or PET scan ( 5 due to clinical deterioration, 3 scheduling or technical difficulties) were excluded from analysis. Of the 29 patients with baseline and postchemotherapy ${ }^{18} \mathrm{~F}-\mathrm{FDG}$ PET and CT scans potentially suitable for analysis, 6 had talc pleurodesis within 6 mo of the baseline ${ }^{18} \mathrm{~F}-\mathrm{FDG}$ PET scan.

Therefore, 23 patients were eligible and had data suitable for analysis. There were 3 women and 20 men, and the mean age was $65 \mathrm{y}$ (range, 52-75 y). 


\section{CT Scans}

Of the 23 patients, 20 had measurable disease at baseline, and 3 had nonmeasurable disease on CT. Of the 20 patients with measurable disease, 7 achieved a PR after 1 cycle of chemotherapy and 13 had SD (Table 1). All of the patients with a PR after 1 cycle of chemotherapy had the response confirmed with a repeat $\mathrm{CT}>4$ wk later. No patients had disease progression after 1 cycle of therapy. Of the 13 patients with SD after 1 cycle, 3 subsequently achieved a PR with further chemotherapy. An example of the CT measurements applied is shown in Figure 2.

\section{${ }^{18}$ F-FDG PET Quantitative Analysis}

In 22 of the 23 patients, a VOI was successfully generated using the semiautomated region-growing algorithm. These VOIs appeared visually representative of the tumor region in all 22 patients. Figure 3 demonstrates an example of the VOI generated before and after chemotherapy using the region-growing algorithm. In 1 patient, with radiologically nonmeasurable disease, there was minimal ${ }^{18} \mathrm{~F}-\mathrm{FDG}$-avid pleural disease, and the algorithm was unable to generate a tumor volume. This tumor was regarded as nonevaluable by PET. The baseline TGV values ranged from 278 to 16,101 $\mathrm{SUV} \cdot \mathrm{mL}$, reflecting the large variation in tumor bulk on metabolic imaging.

Among the 20 patients with CT-measurable disease, the changes in TGV and SUVmax as a percentage of baseline value after 1 cycle of chemotherapy are shown against changes in the summated unidimensional measurement of tumor on CT in Figure 4. The results are displayed according to the radiological response status after 1 cycle of chemotherapy.

For the 7 patients who attained a radiological PR after 1 cycle of chemotherapy, the median TGV after chemotherapy was $30 \%$ of the baseline value (range, $11 \%-71 \%$ ). In the 13 patients with radiologically $\mathrm{SD}$, the median TGV after chemotherapy was $71 \%$ of the baseline value (range, 8\%-113\%) (Table 1). The corresponding TGV measurements show a greater percentage decline among those with PR compared with CT measurements but, more importantly, show a greater spread of data among those with SD (Fig. 4). The Pearson correlation coefficient for the percentage change in TGV compared with the percentage change in CT was $0.69(P<0.001, n=20)$.

SUVmax measurements were also compared with CT measurements (Table 1; Fig. 4). In the CT responding patients the SUVmax fell to a median of $59 \%$ of the pretreatment value (range, 39\%-72\%) and in the patients with CT SD the median SUVmax was $80 \%$ of the baseline value (range, 40\%-111\%). The Pearson correlation coefficient for the percentage change in SUVmax compared with the percentage change in CT was $0.55(P=0.011, n=20)$. The changes in SUVmax values demonstrate a narrower dynamic range than the change in TGV values for both $\mathrm{CT}$ responding and stable patients.

Table 2 lists all of the baseline and postchemotherapy TGV and SUVmax values for the patient group, arranged according to CT response.

TABLE 1

Patient Demographics and ${ }^{18}$ F-FDG PET Response of the 23 Patients in the Study, with Patients Grouped According to CT Response Attained After 1 Cycle of Chemotherapy

\begin{tabular}{|c|c|c|c|}
\hline \multirow[b]{2}{*}{ Patient demographics } & \multicolumn{3}{|c|}{ CT response } \\
\hline & PR & SD & $\mathrm{nm}$ \\
\hline No. of patients (\%) & $7(30)$ & $13(57)$ & $3(13)$ \\
\hline \multicolumn{4}{|l|}{ Age $(y)$} \\
\hline Median & 67 & 64 & 67 \\
\hline Range & $61-71$ & $54-75$ & $52-73$ \\
\hline Male sex (\%) & $5(71)$ & $12(92)$ & $3(100)$ \\
\hline \multicolumn{4}{|l|}{ Baseline TGV (SUV.mL) } \\
\hline Median & 3,372 & 5,056 & $844^{*}$ \\
\hline Range & $278-16,101$ & $897-15,810$ & $834-853$ \\
\hline \multicolumn{4}{|l|}{ Baseline SUVmax (SUV) } \\
\hline Median & 12.2 & 12.8 & $6.4^{\star}$ \\
\hline Range & $5.4-13.9$ & $5.5-18.1$ & $5.3-7.5$ \\
\hline \multicolumn{4}{|l|}{ TGV1/TGV0 (\%) ${ }^{\dagger}$} \\
\hline Median & 30 & 71 & $92^{*}$ \\
\hline Range & $11-71$ & $8-113$ & $83-101$ \\
\hline \multicolumn{4}{|l|}{ SUVmax1/SUVmax0 (\%) } \\
\hline Median & 59 & 80 & $98^{\star}$ \\
\hline Range & $39-72$ & $40-111$ & $92-103$ \\
\hline $\begin{array}{l}{ }^{*} \mathrm{~A} \text { TGV or SUVmax meas } \\
\text { oints. } \\
{ }^{\dagger} \text { TGV after } 1 \text { cycle of che } \\
{ }^{\ddagger} \text { SUVmax after } 1 \text { cycle of } \\
\text { PR = partial response; }\end{array}$ & $\begin{array}{l}\text { ned in only } 20 \\
\text { aseline TGV v } \\
\text { by baseline SI } \\
=\text { not measur }\end{array}$ & $\begin{array}{l}\text {, median and } \\
\text { r a percentage } \\
\text { ressed as a pe }\end{array}$ & n only 2 data \\
\hline
\end{tabular}




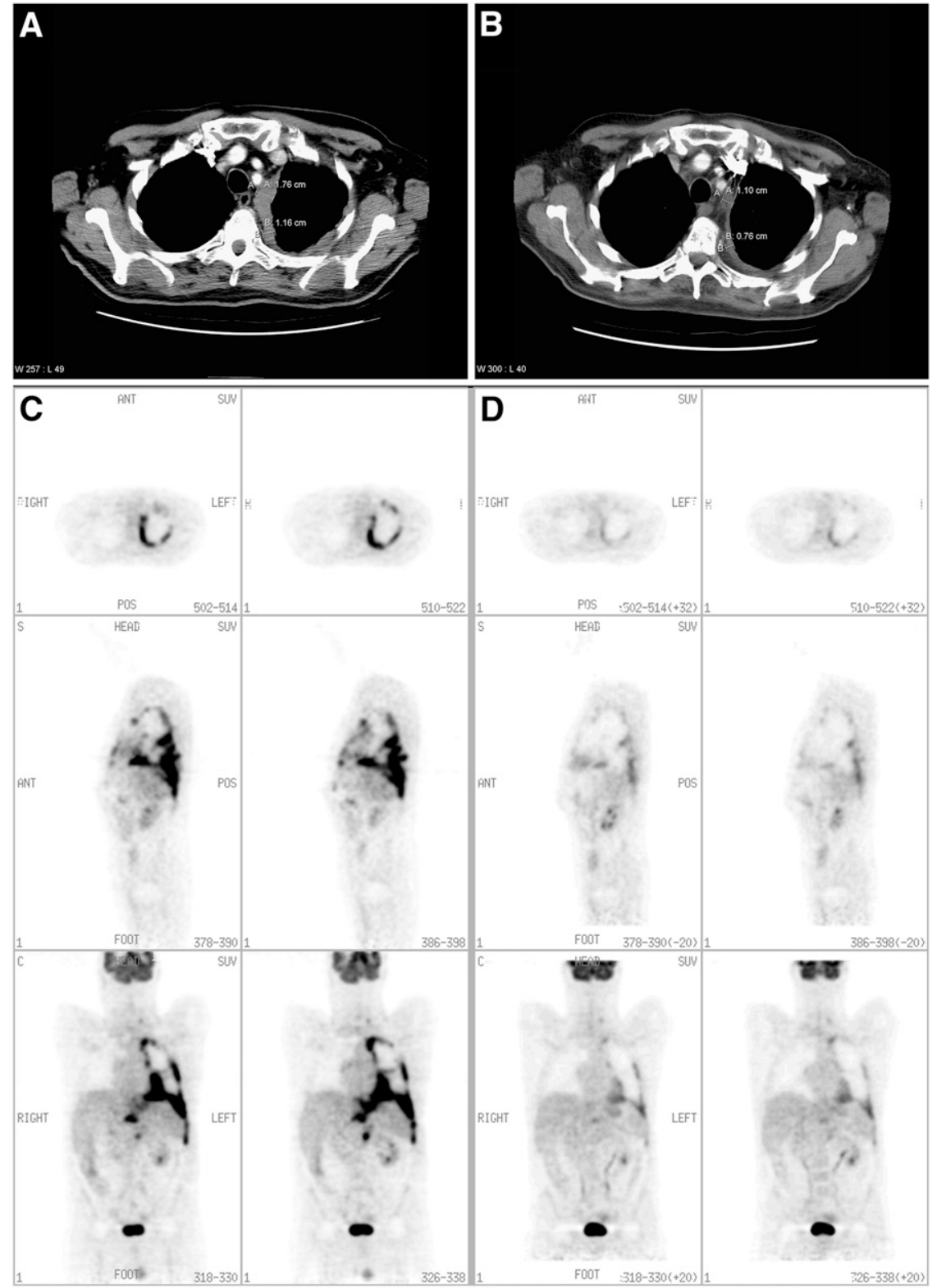

FIGURE 2. Representative CT transaxial slices of a patient with mesothelioma (A) before chemotherapy and (B) after chemotherapy. Measurements according to modified RECIST criteria have been applied. The patient had a radiological partial response after 1 cycle of chemotherapy. The challenge of defining a measurement site to determine response is demonstrated. Representative ${ }^{18} \mathrm{~F}$ FDG PET transverse, sagittal, and coronal slices in the same patient $(C)$ before chemotherapy and (D) after 1 cycle of chemotherapy. A significant reduction in intensity and extent of ${ }^{18} \mathrm{~F}$-FDG uptake in the left pleural cavity is demonstrated. The response is more clearly visualized on the ${ }^{18} \mathrm{~F}-\mathrm{FDG}$ PET imaging, and the degree of change compared with baseline in the patient was greater (TGV fell to $11 \%$ of baseline on the postchemotherapy scan, compared with a fall to $63 \%$ of baseline on CT measurements).

\section{Survival}

At the time of censorship, 11 of 23 patients were alive, and the median survival of the overall group was 9 mo.

Cox regression analysis demonstrated that the survival of the 7 patients who achieved a PR by CT scan after 1 cycle of chemotherapy was not statistically superior to that of the remaining patients $(P=0.38)$ and the percentage change in CT measurements after 1 cycle of chemotherapy did not significantly predict survival $(P=0.131, n=20)$.

The ${ }^{18}$ F-FDG PET analysis demonstrated that the percentage change in SUVmax with chemotherapy was also not predictive of patient survival $(P=0.097, n=22)$. However, the percentage change in TGV after chemotherapy was strongly predictive of survival $(P=0.015, n=22)$. The hazard ratio for a $10 \%$ change in TGV after chemotherapy was 0.64 (95\% confidence interval [CI], 0.26-0.94), indi- cating that a $10 \%$ reduction in $\mathrm{TGV}$ after chemotherapy was associated in our study patients with a $36 \%$ reduction in the risk of death (Table 3).

Exclusion of the 2 patients with prior talc pleurodesis (7 and 9 mo before commencement of chemotherapy) from the Cox regression analysis results in a hazard ratio of 0.70 for a $10 \%$ reduction in TGV $(95 \% \mathrm{CI}, 0.58-0.90 ; P=0.008$, $n=20$ ). Therefore, despite the loss of some statistical power, there remains a significant relationship between the reduction in TGV and survival.

The Kaplan-Meier analysis (Fig. 5) illustrates the significant relationship between the magnitude of TGV percentage change and survival ( $\log$ rank statistic, $11.24 ; P=0.0036)$. The survival of those whose TGV after 1 cycle of chemotherapy fell to $<60 \%$ of baseline was superior to that of those with a smaller change (hazard ratio, $0.22 ; 95 \% \mathrm{CI}, 0.09-0.86 ; P=0.03$ ). 

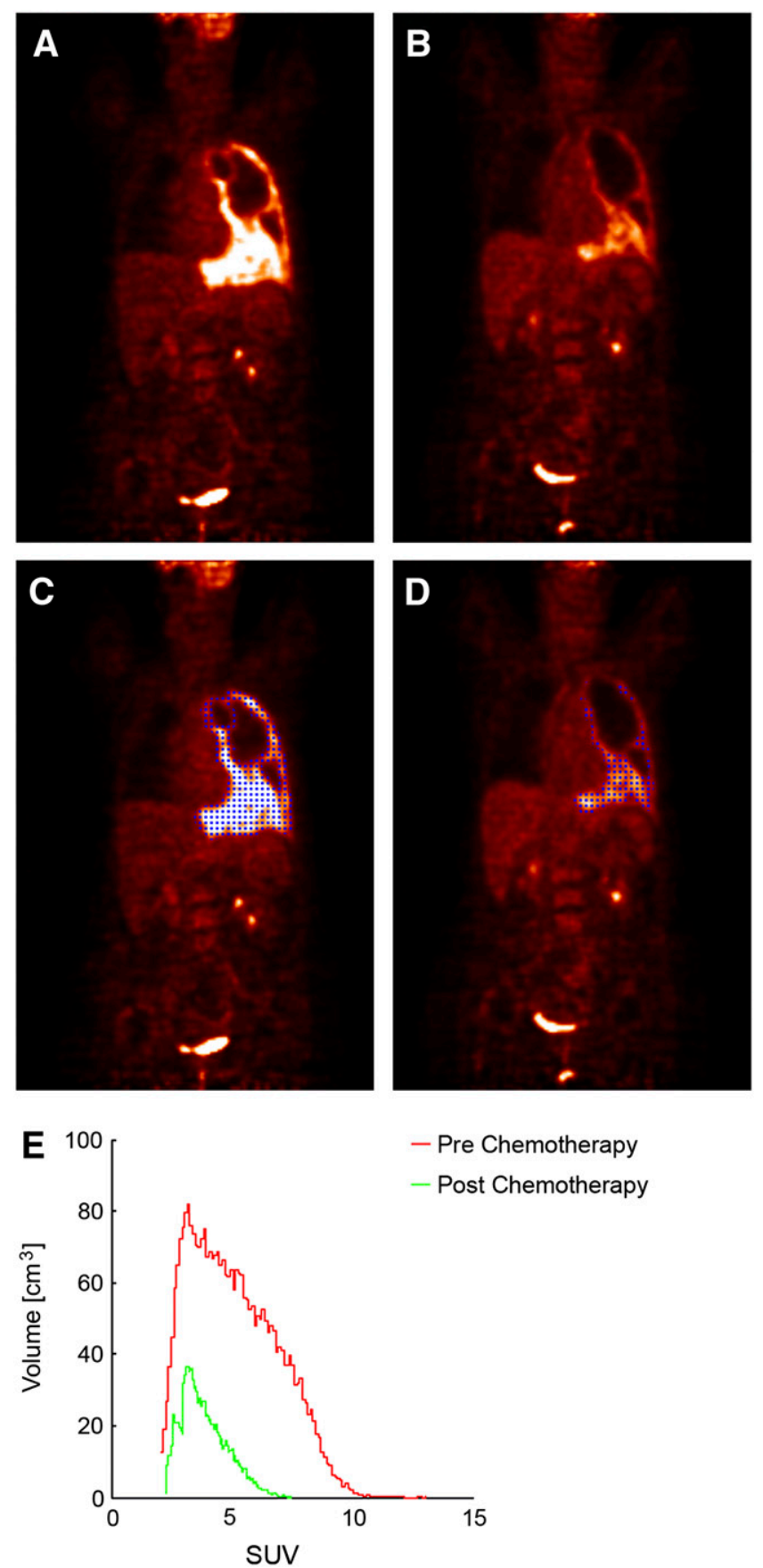

FIGURE 3. Representative ${ }^{18} \mathrm{~F}-\mathrm{FDG}$ PET coronal slices in a patient with left pleural mesothelioma $(A)$ before chemotherapy and $(B)$ after 1 cycle of chemotherapy, demonstrating reduction in the extent and intensity of ${ }^{18} \mathrm{~F}-\mathrm{FDG}$ activity. The region generated by the semiautomated region-growing algorithm is shown on the coronal slice (C) before chemotherapy and (D) after chemotherapy. (A-D) illustrate one representative coronal slice both before and after chemotherapy; however, in practice the region is grown in 3 dimensions to define an overall volume of interest (VOI). (E) Histogram of the SUV voxel values of the VOI generated by the region-growing algorithm in this patient before chemotherapy (red line) and after chemotherapy (green line). The histogram demonstrates both a reduction in the numerical SUV values and in the overall volume of metabolically active tumor. The TGV fell to $30 \%$ of the prechemotherapy value.

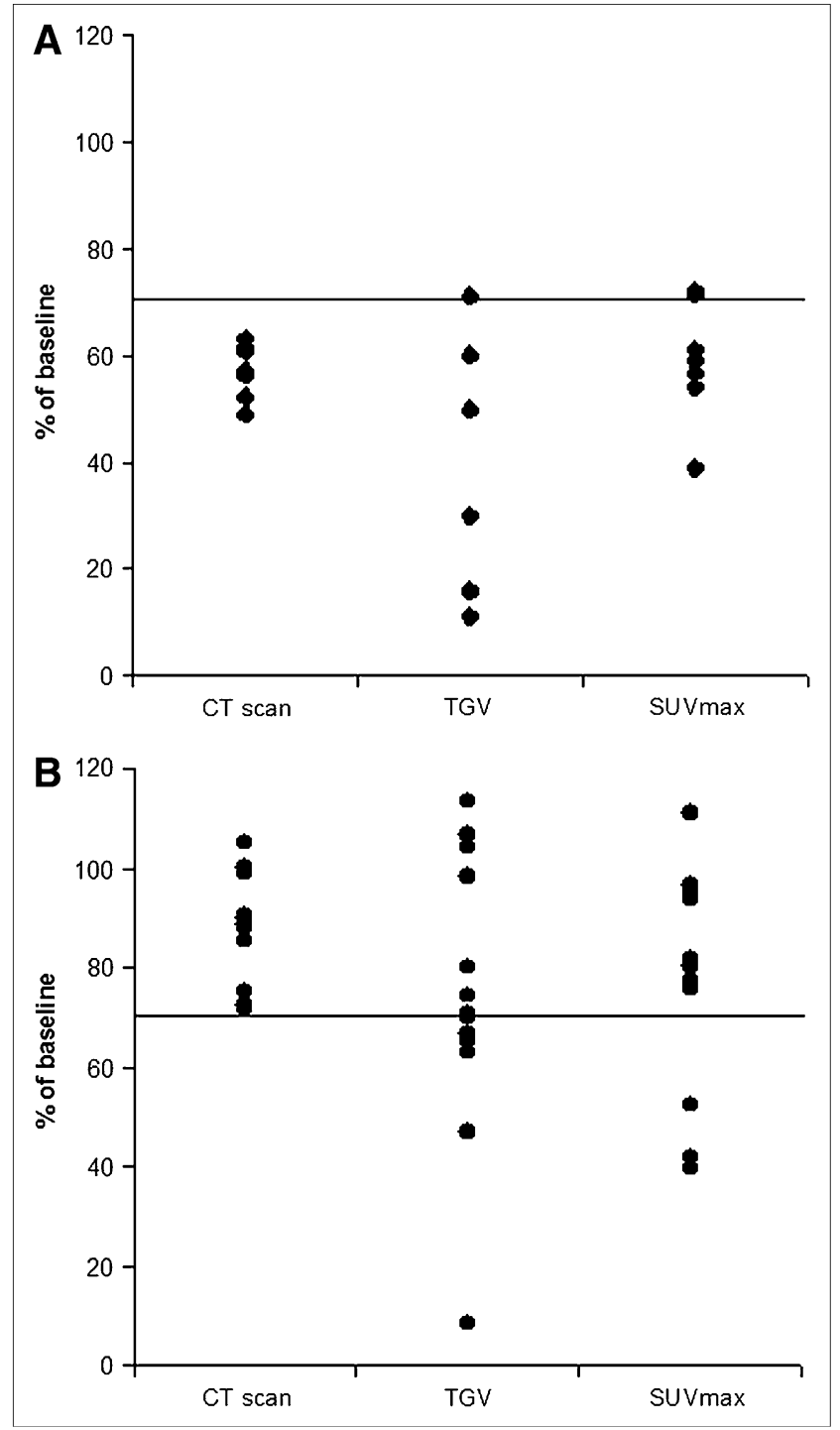

FIGURE 4. ${ }^{18} \mathrm{~F}-\mathrm{FDG}$ PET TGV and SUVmax percentage response values compared with CT response values in the 7 patients with CT-defined PR $(A)$ and 13 patients with CT-defined $\mathrm{SD}$ after 1 cycle of chemotherapy (B). All values are expressed as a percentage of the baseline value. The solid line represents the $70 \%$ value used in CT to define a PR.

The survival of patients whose TGV increased after chemotherapy was compared with those whose TGV decreased with therapy. The median survival in the 4 patients who had an increase in TGV after 1 cycle of chemotherapy was 4.9 mo (95\% CI, 0.0-10.4) compared with 11.5 mo (95\% CI, 7.1-15.9) in the 18 patients whose TGV fell after chemotherapy (log rank statistic, $2.89 ; P=0.09$ ). This is not statistically significant because of the small sample size; however, further investigation in a larger patient group is warranted.

\section{DISCUSSION}

This study introduces a novel method of semiautomatically defining tumor volumes on ${ }^{18}$ F-FDG PET using software that applies a mathematic algorithm to delineate tumor 
TABLE 2

Baseline and After 1 Cycle of Chemotherapy, TGV and SUVmax Values for All Patients, Grouped According to CT Response

\begin{tabular}{|c|c|c|c|c|c|c|c|c|c|}
\hline Status & Sex & Age (y) & TGVO & TGV1 & TGV1/TGV0 (\%) & SUVO & SUV1 & SUV1/SUV0 (\%) & Survival (mo) \\
\hline \multicolumn{10}{|l|}{ CT PR } \\
\hline 1 & $M$ & 69 & 278 & 138 & 50 & 5.6 & 4.0 & 71 & $23.7^{\dagger}$ \\
\hline 2 & $M^{*}$ & 67 & 1,100 & 177 & 16 & 13.9 & 7.5 & 54 & 8.0 \\
\hline 3 & $\mathrm{~F}$ & 61 & 16,101 & 4,814 & 30 & 13.2 & 7.5 & 57 & $18.7^{\dagger}$ \\
\hline 4 & $M$ & 69 & 7,205 & 1,130 & 16 & 11.5 & 8.3 & 72 & 11.5 \\
\hline 5 & M & 71 & 9,840 & 1,089 & 11 & 13.1 & 5.1 & 39 & $10.3^{\dagger}$ \\
\hline 6 & $M$ & 66 & 3,372 & 2,033 & 60 & 12.2 & 7.4 & 61 & $3.9^{\dagger}$ \\
\hline 7 & $\mathrm{~F}$ & 65 & 549 & 390 & 71 & 5.4 & 3.2 & 59 & $4.4^{\dagger}$ \\
\hline \multicolumn{10}{|l|}{ CT SD } \\
\hline 8 & M & 68 & 9,177 & 10,412 & 113 & 12.8 & 12.2 & 95 & 4.9 \\
\hline 9 & $M$ & 55 & 4,701 & 5,012 & 107 & 5.5 & 4.5 & 82 & 1.4 \\
\hline 10 & $M$ & 63 & 5,139 & 5,057 & 98 & 9.0 & 8.7 & 97 & 6.9 \\
\hline 11 & $M$ & 68 & 3,048 & 2,268 & 74 & 18.1 & 14.0 & 77 & 9.5 \\
\hline 12 & $M$ & 54 & 5,631 & 3,762 & 67 & 9.0 & 6.8 & 76 & 17.6 \\
\hline 13 & $M$ & 64 & 4,398 & 2,862 & 65 & 13.0 & 6.8 & 52 & 5.9 \\
\hline 14 & $M^{*}$ & 60 & 15,809 & 11,104 & 70 & 13.6 & 11.1 & 82 & $14.5^{\dagger}$ \\
\hline 15 & $M$ & 55 & 3,104 & 265 & 8 & 9.3 & 3.7 & 40 & $12.4^{\dagger}$ \\
\hline 16 & $\mathrm{~F}$ & 71 & 1,413 & 1,470 & 104 & 13.2 & 10.6 & 80 & 9.9 \\
\hline 17 & $M$ & 74 & 5,484 & 3,440 & 63 & 10.9 & 10.2 & 94 & $6.2^{\dagger}$ \\
\hline 18 & $M$ & 65 & 9,635 & 7,754 & 80 & 13.0 & 10.4 & 80 & 5.9 \\
\hline 19 & $M$ & 75 & 897 & 423 & 47 & 17.8 & 7.5 & 42 & $2.9^{\dagger}$ \\
\hline 20 & $M$ & 54 & 5,056 & 3,574 & 71 & 10.0 & 11.1 & 111 & $2.5^{\dagger}$ \\
\hline \multicolumn{10}{|c|}{ CT nonmeasurable } \\
\hline 21 & M & 52 & 853 & 861 & 101 & 5.3 & 4.9 & 92 & 7.5 \\
\hline 22 & $M$ & 73 & 834 & 689 & 83 & 7.5 & 7.7 & 103 & 12.2 \\
\hline 23 & $M$ & 67 & $\mathrm{n} / \mathrm{e}$ & $\mathrm{n} / \mathrm{e}$ & $n / e$ & $\mathrm{n} / \mathrm{e}$ & $\mathrm{n} / \mathrm{e}$ & $n / e$ & $8.4^{\dagger}$ \\
\hline
\end{tabular}

${ }^{*}$ Previous talc pleurodesis.

tSurvival at time of censorship (alive).

TGV0 = TGV at baseline (before chemotherapy); TGV1 = TGV after 1 cycle of chemotherapy; TGV1/TGV0 (\%) = TGV after 1 cycle of chemotherapy divided by baseline TGV value and expressed as a percentage; SUVO = SUVmax at baseline (before chemotherapy); SUV1 = SUVmax after 1 cycle of chemotherapy; SUV1/SUVO (\%) = SUVmax after 1 cycle of chemotherapy divided by baseline SUVmax value and expressed as a percentage; $\mathrm{n} / \mathrm{e}=$ not evaluable, as a VOI could not be generated on PET images.

regions. Mesothelioma, as a predominantly contiguous tumor encasing the pleural space, was ideally suited to a semiautomated approach, as defining the tumor boundaries by hand would have been laborious and prone to error. This study demonstrates that the region-growing algorithm is feasible, resulting in a visually representative tumor VOI in 22 of 23 patients. It included 2 patients whose tumor bulk was

TABLE 3

Cox Proportional Regression Analysis of Relationship Between Change in CT, SUVmax, and TGV after 1 Cycle of Chemotherapy and Survival

\begin{tabular}{lccc}
\hline 10\% change & Hazard ratio & $95 \% \mathrm{Cl}$ & $P$ value \\
\hline CT & 0.71 & $0.21-1.07$ & 0.131 \\
SUVmax & 0.65 & $0.12-1.05$ & 0.097 \\
TGV & 0.64 & $0.26-0.94$ & $\mathbf{0 . 0 1 5}$
\end{tabular}

The boldface $P$ value 0.015 indicates the value is statistically significant. too small to be measurable on the CT scan. The differentiation between tumor and background on ${ }^{18} \mathrm{~F}-\mathrm{FDG}$ PET was insufficient for region generation in only 1 patient.

Volume-based and semiautomated methods of CT scan analysis for patients with mesothelioma are being developed $(18,19) .{ }^{18} \mathrm{~F}-\mathrm{FDG}$ PET, however, is a functional measure of tumor metabolic activity, which is potentially more sensitive in detecting change to therapy than anatomic measurements. ${ }^{18}$ F-FDG PET has been demonstrated to be useful in the prediction of response to chemotherapy in several solid tumors, with the reduction in ${ }^{18} \mathrm{~F}$-FDG uptake often preceding radiological changes (4-6,20-22). There are also emerging survival data with metabolic responses associated with a survival advantage in lung, breast, esophageal, and pancreatic cancer $(5-7,21,23)$.

Volume-based ${ }^{18}$ F-FDG PET tumor assessment has been performed in lung cancer (24), colorectal cancer (25), and glioma (26). Most published methods used fixed threshold techniques. Fixed threshold techniques are poorly suited to mesothelioma because of the plaquelike tumor mass and the problems associated with differentiating tumor from 


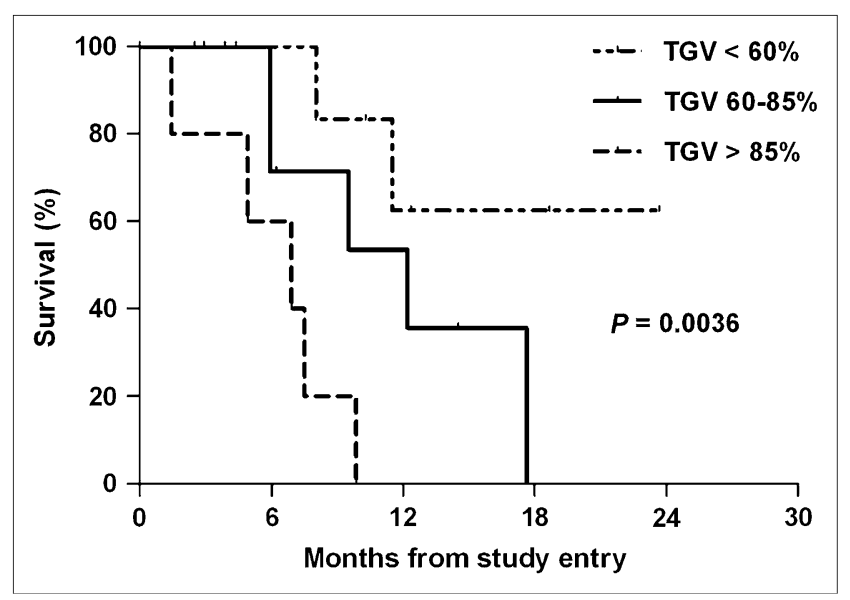

FIGURE 5. Kaplan-Meier survival curves illustrate the relationship between the degrees of reduction in TGV compared with baseline and survival. TGV $<60 \%$ represents a reduction in TGV after 1 cycle of chemotherapy to less than $60 \%$ of the baseline value. TGV $60 \%-85 \%$ represents a reduction to $60 \%-$ $85 \%$ of the baseline value. TGV $>85 \%$ includes patients whose TGV after chemotherapy was $85 \%$ or greater than the baseline value and patients whose TGV increased after chemotherapy.

adjacent normal tissue, such as chest wall, mediastinum, liver, and heart. By using iterative thresholding and taking into account the ${ }^{18} \mathrm{~F}-\mathrm{FDG}$ activity in surrounding normal tissues, our semiautomated region-growing algorithm was capable of delineating tumor boundaries. The VOI generated from the region-growing algorithm was then used to obtain a measure of tumor glycolysis, which we termed TGV. This measure is similar to TLG (total lesion glycolysis) developed by Larson et al. (24). Although the method of generating the tumor volume differs between the 2 studies, the concept of assessing total glycolysis within viable tumor is similar.

Despite the small sample size in our study, statistical analysis by Cox regression demonstrates a strong relationship between the change in TGV after 1 cycle of chemotherapy and the survival for patients with mesothelioma. Neither SUVmax nor CT response after 1 cycle of chemotherapy, however, predicted survival. Not only are the hazard ratios superior for TGV in comparison with CT and SUVmax, but also the wider dynamic range of change in TGV (range, 11\%-113\%) compared with change in SUVmax (range, 39\%-111\%) or CT (range, 49\%-105\%) allows for potentially better stratification of response. The Kaplan-Meier analysis further supports the value of TGV measurements and illustrates a continuum in the relationship between decrease in TGV and survival.

A recent paper by Ceresoli et al. (27) assessed response to chemotherapy in mesothelioma with ${ }^{18} \mathrm{~F}$-FDG PET. The authors defined a $25 \%$ decrease in SUVmax as a metabolic response. They were able to show a relationship between metabolic response and time to progression and a trend to improved survival. SUVmax is a single-pixel value, which reflects maximum intensity of ${ }^{18} \mathrm{~F}-\mathrm{FDG}$ activity in the tumor. TGV is a 3D measure and incorporates both tumor volume and metabolic activity. Therefore, TGV is reflective of change throughout the entire tumor mass and may potentially be a more sensitive method of detecting change than a single-pixel value. This may explain the statistically significant relationship between change in TGV and survival that was demonstrated in our study, whereas change in SUVmax and survival was not significant in the Ceresoli study and our experience.

There was considerable overlap in the change in TGV values in the group of patients with CT PR and those with CTdefined SD. The majority of patients in our study had SD on CT after 1 cycle of chemotherapy; however, there are several patients in the CT-defined SD group whose TGV fell to the same extent as those who were defined by $\mathrm{CT}$ as attaining a PR. This demonstrates the limited sensitivity of the modified RECIST criteria and highlights the difficulties of applying the principles of unidimensional response measurements to a tumor that encases the pleural cavity (28). Defining the site of measurement by modified RECIST is challenging, and obtaining a PR is dependent on the thickness of involved pleura decreasing by $30 \%$. The well-recognized difficulties associated with CT response assessment in mesothelioma highlight the need for a more reliable and sensitive method of response assessment in this tumor type.

The attrition of patients who are not well is a potential limitation of this study, providing less data in the nonresponding group. Five patients were unable to complete either 1 course of chemotherapy or the pre- and postchemotherapy ${ }^{18}$ F-FDG PET and CT scans. In clinical practice, these patients likely stop chemotherapy based on clinical assessment, independent of imaging response. Six patients initially recruited to this study had talc pleurodesis performed within 6 mo of study entry. The inflammatory reaction to talc pleurodesis is reported to persist on ${ }^{18} \mathrm{~F}-\mathrm{FDG}$ PET for months to years (15). However, this reaction is likely to be most intense close to the time of the pleurodesis. We included in this study patients in whom pleurodesis was performed $>6$ mo earlier, as any change in tumor ${ }^{18} \mathrm{~F}-\mathrm{FDG}$ uptake due to chemotherapy was likely to be greater than residual change in ${ }^{18} \mathrm{~F}-\mathrm{FDG}$ uptake from pleurodesis. Therefore, 2 analyzed patients had a pleurodesis 7 and 9 mo before chemotherapy. Both patients had a fall in TGV to $16 \%$ and $70 \%$ of baseline, respectively. Excluding these patients did not reduce the significance of the relationship between change in TGV and survival. The use of ${ }^{18} \mathrm{~F}-\mathrm{FDG}$ PET for tumor response evaluation in patients who have had previous pleurodesis, however, needs further clarification.

We have not attempted to define absolute criteria for "TGV response" in this study. Further clinical data need to be obtained before a value representing an unequivocal decrease in TGV can be proposed. This article, however, demonstrates the feasibility and potential clinical utility of volume-based PET measures such as TGV in assessing response to chemotherapy. A reduction in TGV after 1 cycle of chemotherapy correlated well with CT response and, importantly, had greater prognostic significance than that demonstrated with SUVmax or CT scan measurements. In combination with the Ceresoli 
experience (27), this work supports the utility of ${ }^{18} \mathrm{~F}$-FDG PET in response assessment in malignant pleural mesothelioma and suggests that a metabolic response to chemotherapy is of prognostic significance in this complex tumor type.

\section{CONCLUSION}

Serial ${ }^{18}$ F-FDG PET in mesothelioma appears useful in predicting response and patient survival after only 1 cycle of chemotherapy. TGV, a volume-based measure of total glycolysis, was superior to SUVmax and to CT measurements in predicting survival in the patient group studied. Therefore, metabolic imaging has the potential to improve the care of patients receiving chemotherapy for mesothelioma by the early identification of responding patients. This technology may also be useful in the assessment of new systemic treatments for mesothelioma.

\section{APPENDIX}

The semiautomated region-growing algorithm is a mathematically defined iterative algorithm that uses an adaptive threshold to delineate 3D tumor boundaries. The operator chooses a seed-point anywhere in the visible tumor region. The tumor region grows contiguously from the seed-point to include any neighboring voxels with counts/voxel above the current threshold value. The "threshold value" is recalculated after each iteration using the formula: Threshold value $=$ threshold factor $\cdot$ current mean.

The mean is recalculated after each iteration and the threshold value is reapplied. This process continues until no further voxels can be drawn. To ensure reproducibility, once the final volume is defined, the region is regrown from the voxel with the maximum counts/voxel, outward. This ensures that wherever the seed-point is placed within the tumor, the same region will always be grown.

The "threshold factor" is a function of the maximum normal level (MNL) that is set by the user. The threshold factor varies depending on the current mean SUV of the growing region and the MNL value and has range of values between 0 and 1 . The threshold factor approaches 1.0 when the mean SUV in the growing region is approaching the MNL. However, when the growing-region mean SUV is much higher than the MNL, the threshold factor reduces to a minimum approaching 0 .

The "MNL" is derived from a region of interest placed in normal background tissue and is defined as the mean background region SUV +3 SDs.

The software was programmed in Interactive Data Language (IDL) and is not commercially available. Release of the program to several sites for research purposes has been performed in a cooperative "shareware" arrangement.

\section{ACKNOWLEDGMENTS}

This study was supported by a research grant from the Cancer Council of Western Australia. We gratefully acknowl- edge the efforts of Judy Innes-Rowe, Dr. Karen Tucker, and the WA PET Centre and Cyclotron staff.

\section{REFERENCES}

1. Byrne MJ, Nowak AK. Modified RECIST criteria for assessment of response in malignant pleural mesothelioma. Ann Oncol. 2004;15:257-260.

2. Nowak AK, Byrne MJ, Williamson R, et al. A multicentre phase II study of cisplatin and gemcitabine for malignant mesothelioma. Br J Cancer. 2002;87: 491-496.

3. Nowak AK, Stockler MR, Byrne MJ. Assessing quality of life during chemotherapy for pleural mesothelioma: feasibility, validity, and results of using the European Organization for Research and Treatment of Cancer Core Quality of Life Questionnaire and Lung Cancer Module. J Clin Oncol. 2004;22:31723180 .

4. Wahl RL, Zasadny K, Helvie M, Hutchins GD, Weber B, Cody R. Metabolic monitoring of breast cancer chemohormonotherapy using positron emission tomography: initial evaluation. J Clin Oncol. 1993;11:2101-2111.

5. Weber WA, Ott K, Becker K, et al. Prediction of response to preoperative chemotherapy in adenocarcinomas of the esophagogastric junction by metabolic imaging. J Clin Oncol. 2001;19:3058-3065.

6. Weber WA, Petersen V, Schmidt B, et al. Positron emission tomography in nonsmall-cell lung cancer: prediction of response to chemotherapy by quantitative assessment of glucose use. J Clin Oncol. 2003;21:2651-2657.

7. Dose Schwarz J, Bader M, Jenicke L, Hemminger G, Janicke F, Avril N. Early prediction of response to chemotherapy in metastatic breast cancer using sequential ${ }^{18}$ F-FDG PET. J Nucl Med. 2005;46:1144-1150.

8. Benard F, Sterman D, Smith RJ, Kaiser LR, Albelda SM, Alavi A. Metabolic imaging of malignant pleural mesothelioma with fluorodeoxyglucose positron emission tomography. Chest. 1998;114:713-722.

9. Benard F, Sterman D, Smith RJ, Kaiser LR, Albelda SM, Alavi A. Prognostic value of FDG PET imaging in malignant pleural mesothelioma. J Nucl Med. 1999;40:1241-1245.

10. Flores RM, Akhurst T, Gonen M, Larson SM, Rusch VW. Positron emission tomography defines metastatic disease but not locoregional disease in patients with malignant pleural mesothelioma. J Thorac Cardiovasc Surg. 2003;126:11-16.

11. Shankar LK, Hoffman JM, Bacharach S, et al. Consensus recommendations for the use of ${ }^{18} \mathrm{~F}$-FDG PET as an indicator of therapeutic response in patients in National Cancer Institute trials. J Nucl Med. 2006;47:1059-1066.

12. Keyes JW. SUV: standard uptake or silly useless value? J Nucl Med. 1995;36: 1836-1839.

13. Hamberg LM, Hunter GJ, Alpert NM, Choi NC, Babich JW, Fischman AJ. The dose uptake ratio as an index of glucose metabolism: useful parameter or oversimplification? J Nucl Med. 1994;35:1308-1312.

14. Young H, Baum R, Cremerius U, et al. Measurement of clinical and subclinical tumour response using $\left[{ }^{18} \mathrm{~F}\right]$-fluorodeoxyglucose and positron emission tomography: review and 1999 EORTC recommendations-European Organization for Research and Treatment of Cancer (EORTC) PET Study Group. Eur J Cancer. 1999;35:1773-1782.

15. Kwek BH, Aquino SL, Fischman AJ. Fluorodeoxyglucose positron emission tomography and CT after talc pleurodesis. Chest. 2004;125:2356-2360.

16. Green AJ, Baig S, Francis RJ, Begent RHJ. Semi quantitative analysis of F18FDG imaging using a hybrid camera for the assessment of tumor response to therapy [abstract]. Eur J Nucl Med. 2001;28(suppl):S132.

17. Boucek J, Francis RJ, Green AJ. Automated approach to identification and quantitation of tumour volumes in chemotherapy monitoring using FDG PET [abstract]. J Nucl Med. 2005;46(suppl):464P.

18. Armato SG III, Oxnard GR, MacMahon H, et al. Measurement of mesothelioma on thoracic CT scans: a comparison of manual and computer-assisted techniques. Med Phys. 2004;31:1105-1115.

19. Steinert HC, Santos Dellea MM, Burger C, Stahel R. Therapy response evaluation in malignant pleural mesothelioma with integrated PET-CT imaging. Lung Cancer. 2005;49:S33-S35.

20. Smith IC, Welch AE, Hutcheon AW, et al. Positron emission tomography using $\left[{ }^{18} \mathrm{~F}\right]$-fluorodeoxy-D-glucose to predict the pathologic response of breast cancer to primary chemotherapy. J Clin Oncol. 2000;18:1676-1688.

21. Maisey NR, Webb A, Flux GD, et al. FDG-PET in the prediction of survival of patients with cancer of the pancreas: a pilot study. Br J Cancer. 2000;83:287293.

22. Jansson T, Westlin JE, Ahlstrom H, Lilja A, Langstrom B, Bergh J. Positron emission tomography studies in patients with locally advanced and/or metastatic breast cancer: a method for early therapy evaluation? J Clin Oncol. 1995;13: 1470-1477. 
23. Flamen P, Van CE, Lerut A, et al. Positron emission tomography for assessment of the response to induction radiochemotherapy in locally advanced oesophageal cancer. Ann Oncol. 2002;13:361-368.

24. Larson SM, Erdi Y, Akhurst T, et al. Tumor treatment response based on visual and quantitative changes in global tumor glycolysis using PET-FDG imaging: the visual response score and the change in total lesion glycolysis. Clin Positron Imaging. 1999;2:159-171.

25. Guillem JG, Moore HG, Akhurst T, et al. Sequential preoperative fluorodeoxyglucose-positron emission tomography assessment of response to preoperative chemoradiation: a means for determining longterm outcomes of rectal cancer. J Am Coll Surg. 2004;199:1-7.
26. Tralins KS, Douglas JG, Stelzer KJ, et al. Volumetric analysis of ${ }^{18}$ F-FDG PET in glioblastoma multiforme: prognostic information and possible role in definition of target volumes in radiation dose escalation. J Nucl Med. 2002;43:16671673.

27. Ceresoli GL, Chiti A, Zucali PA, et al. Early response evaluation in malignant pleural mesothelioma by positron emission tomography with $\left[{ }^{18} \mathrm{~F}\right]$ fluorodeoxyglucose. J Clin Oncol. 2006;24:4587-4593.

28. van Klaveren RJ, Aerts JG, de Bruin H, Giaccone G, Manegold C, van Meerbeeck JP. Inadequacy of the RECIST criteria for response evaluation in patients with malignant pleural mesothelioma. Lung Cancer. 2004;43: 63-69. 